\title{
THE SOLUTION OF A DIOPHANTINE EQUATION
}

\author{
CHARLES P. BENNER
}

Solutions of the diophantine equation ${ }^{1}$

$$
\prod_{i=1}^{4} \sum_{j=1}^{3} a_{i j} x_{j}=f\left(y_{1}, \cdots, y_{a}\right)
$$

have been given. We generalize the equation by solving the equation

$$
\prod_{i=1}^{2^{n}} \sum_{j=1}^{2^{n}-1} a_{i j} x_{j}=f(y),
$$

in which we suppose that $f(y)=f\left(y_{1}, \cdots, y_{a}\right)$ is a homogeneous polynomial, with integral coefficients, of degree $m$, where $m$ is of the form $2^{p}(2 q+1), q$ being a non-negative integer, $p$ is one of the integers $0,1, \cdots, n-1$, and thus $m \neq 0\left(\bmod 2^{n}\right)$. We suppose further that the rank of the matrix of the forms $\sum_{j=1}^{2^{n}-1} a_{i j} x_{j}\left(i=1, \cdots, 2^{n}\right)$ is $2^{n}-1$ and thus we may choose the notation such that $A$, the determinant of the first $2^{n}-1$ forms, does not vanish. Let $A_{i j}$ be the cofactor of $a_{i j}$ in $A$.

THEOREM. Every integral solution $x_{j}, y_{k}$ of (1) for which the left-hand member does not vanish is equivalent (in a sense to be defined) to a solution given by

$$
\begin{aligned}
& x_{j}=A^{m-1} s^{q} t^{q}\left[t \sum_{r=1}^{2^{n-1}} \alpha_{r} A_{r j}+s \sum_{r=2^{n-1}+1}^{2^{n-1}} \alpha_{r} A_{r j}\right] \quad\left(j=1, \cdots, 2^{n}-1\right), \\
& y_{k}=A^{2^{n} s^{2 n-1-p} t^{2 n-1-p} \beta_{k},}
\end{aligned}
$$

where $s$ and $t$ are given by

$$
\begin{aligned}
& s=\left[\prod_{i=1}^{2^{n}-1} \alpha_{i}\right] \sum_{j=1}^{2^{n}-1} \sum_{r=1}^{2^{n-1}} a_{2^{n} j} \alpha_{r} A_{r j}, \\
& t=A f(\beta)-\left[\prod_{i=1}^{2^{n}-1} \alpha_{i}\right] \sum_{j=1}^{2^{n-1}} \sum_{r=2^{n-1}+1}^{2^{n}-1} a_{2^{n} j} \alpha_{r} A_{r j},
\end{aligned}
$$

the $\alpha$ 's and $\beta$ 's being arbitrary integers.

Proof. If we set

Received by the editors September 27, 1950 and, in revised form, April 20, 1951.

${ }^{1}$ A. A. Aucoin, Solution of a quartic diophantine equation, Boletin Matematico vol. 14 (1941) pp. 36-39. 
(4)

$$
\begin{array}{lr}
\sum_{j=1}^{2^{n}-1} a_{i j} x_{j}=A^{m} \alpha_{i} s^{q} t^{q+1} & \left(i=1, \cdots, 2^{n-1}\right), \\
\sum_{j=1}^{2^{n}-1} a_{i j} x_{j}=A^{m} \alpha_{i} s^{q+1} t^{q} & \left(i=2^{n-1}+1, \cdots, 2^{n}-1\right),
\end{array}
$$

and solve the system of equations for $x_{j}$, we have

$$
x_{j}=A^{m-1} s^{q} t^{q}\left[t \sum_{r=1}^{2^{n-1}} \alpha_{r} A_{r j}+s \sum_{r=2^{n-1}+1}^{2^{n}-1} \alpha_{r} A_{r j}\right] \quad\left(j=1, \cdots, 2^{n}-1\right),
$$

and hence

$$
\sum_{j=1}^{2^{n}-1} a_{2^{n} j} x_{j}=A^{m-1} s^{q} t^{q}\left[t \sum_{j=1}^{2^{n-1}} \sum_{r=1}^{2^{n-1}} a_{2^{n} j \alpha_{r} A_{r j}}\right.
$$

$$
\left.+s \sum_{i=1}^{2^{n}-1} \sum_{r=2^{n-1}+1}^{2^{n-1}} a_{2^{n}} \alpha_{r} A_{r j}\right]
$$

If

$$
y_{k}=A^{2^{n}} s^{2^{n-1-p}} t^{2^{n-1-p}} \beta_{k},
$$

then from (4), (5), and (6) equation (1) becomes

$$
\begin{aligned}
A^{2^{n+p}(2 q+1)-1} s^{2^{n-1}(2 q+1)-1} t^{2^{n-1}(2 q+1)}(P t+Q s) \prod_{i=1}^{2^{n}-1} \alpha_{i} \\
=A^{2^{n+p}(2 q+1)} s^{2^{n-1}(2 q+1)} t^{2^{n-1}(2 q+1)} f(\beta),
\end{aligned}
$$

where

$$
\begin{aligned}
& P=\sum_{j=1}^{2^{n}-1} \sum_{r=1}^{2^{n-1}} a_{2^{n}} \alpha_{r} A_{r j}, \\
& Q=\sum_{j=1}^{2^{n}-1} \sum_{r=2^{n-1}+1}^{2^{n-1}} a_{2^{n}} \alpha_{r} A_{r j} .
\end{aligned}
$$

Now (7) is satisfied identically in the $\alpha$ 's and $\beta$ 's if $s$ and $t$ are given by (3). Hence a solution of (1) is given by (2) if the left-hand member does not vanish. The solution $x_{j}, y_{k}$ is integral if the parameters are integral.

We now define the concept of equivalent solutions. Suppose that $x_{j}=\rho_{j}, y_{k}=\gamma_{k}$ is a solution of the equation

$$
f\left(x_{1}, \cdots, x_{a}\right)=g\left(y_{1}, \cdots, y_{b}\right),
$$

where $f$ and $g$ are homogeneous polynomials with integral coefficients, of degrees $n$ and $m$ respectively. If there are no integers $s>1, \rho_{j}^{\prime}, \gamma_{k}^{\prime}$ 
such that $\rho_{j}=\rho_{j}^{\prime} s^{\lambda}, \gamma_{k}=\gamma_{k}^{\prime} s^{\mu}$, where $\lambda$ and $\mu$ are positive integers such that $\lambda n=\mu m$, then $x_{j}=\rho_{j}, y_{k}=\gamma_{k}$ is defined to be a primitive solution of the given equation. If $x_{j}=\rho_{j}, y_{k}=\gamma_{k}$ is a primitive solution of the given equation and if $\lambda$ and $\mu$ are positive integers such that $\lambda n=\mu m$, then $x_{j}=\rho_{j} t^{\lambda}, y_{k}=\gamma_{k} t^{\mu}$, where $t$ is a nonzero integer, is also a solution and is said to be derived from this primitive solution. Two solutions are said to be equivalent if they may be derived from the same primitive solution.

It remains to be shown that given any solution of (1) which does not cause the left-hand member to vanish, we may choose the parameters in (2) so as to obtain a solution equivalent to the given one. Suppose that $x_{j}=\rho_{j}, y_{k}=\gamma_{k}$ is any such solution of (1). If we choose $\alpha_{i}=\sum_{j=1}^{2^{n}-1} a_{i j} \rho_{j}$ and $\beta_{k}=\gamma_{k}$ then from (1) and (3) we have

$$
\begin{aligned}
s-t= & {\left[\prod_{i=1}^{2^{n}-1} \sum_{j=1}^{2^{n-1}} a_{i j} \rho_{j}\right] \sum_{j=1}^{2^{n}-1} \sum_{r=1}^{2^{n}-1} \sum_{h=1}^{2^{n}-1} a_{2^{n} j} a_{r h} A_{r j \rho_{h}} } \\
& -A\left[\prod_{i=1}^{2^{n}} \sum_{j=1}^{2^{n-1}} a_{i j \rho_{j}}\right] \\
= & {\left[\prod_{i=1}^{2^{n}-1} \sum_{j=1}^{2^{n-1}} a_{i j} \rho_{j}\right]\left[\sum_{j=1}^{2^{n}-1} a_{2^{n}} \sum_{h=1}^{2^{n}-1} \rho_{h} \sum_{r=1}^{2^{n-1}} a_{r h} A_{r j}-A \sum_{j=1}^{2^{n}-1} a_{2^{n} j} \rho_{j}\right] } \\
= & {\left[\prod_{i=1}^{2^{n}-1} \sum_{j=1}^{2^{n}-1} a_{i j}\right]\left[A \sum_{j=1}^{2^{n}-1} a_{2^{n} j \rho_{j}}-A \sum_{j=1}^{2^{n}-1} a_{2^{n} j} \rho_{j}\right]=0 }
\end{aligned}
$$

and therefore $s=t$. Thus (2) becomes

$$
x_{j}=A^{2^{p}(2 q+1)} s^{2 q+1} \rho_{j}, \quad y_{k}=A^{2^{n} s^{2^{n-p}} \gamma_{k}}
$$

which is equivalent to the solution $x_{j}=\rho_{j}, y_{k}=\gamma_{k}$. Hence we may choose the parameters so as to get a solution equivalent to the given solution $x_{j}=\rho_{j}, y_{k}=\gamma_{k}$, provided that the left member of (1) is not zero.

We may also find integers, not all zero, which will cause the lefthand member of (1) to vanish and these together with $y_{k}=0$ will afford additional solutions of (1).

UNIVERSITY OF HOUSTON 